Brief Paper

\title{
EMS Control System Design for a Maglev Vehicle-A Critical System*
}

\section{J. F. WHIDBORNE $\dagger$}

Key Words-Control system design; magnetic suspension; computer-aided design; feedback control; disturbance rejection.

\begin{abstract}
For the effective operation of a magnetically levitated (maglev) vehicle using electro-magnetic suspension, it is necessary that the airgap between the guideway and the levitating magnets is maintained. Such systems, where the output is required to remain strictly within bounds, are known as critical systems. This paper describes the design of the suspension system for a high-speed maglev vehicle which ensures that the airgap is maintained.
\end{abstract}

\section{Introduction}

THE CONCEPT OF a magnetically levitated (maglev) vehicle is not new, but recent advances in technology have made it possible for maglev vehicles to be successfully implemented. The two most effective suspension techniques are the electro-dynamic suspension (EDS) and the electro-magnetic suspension (EMS). EDS systems rely on the fact that a repulsive force is generated when a magnet is moved over a conducting sheet. In order to produce enough force for practical implementation, superconductors are required. Japanese National Railways have made the most progress with this technology, and have tested a system with a 50 -ton vehicle operating at $300 \mathrm{~km} \mathrm{hr}^{-1}$ on a $12.5-\mathrm{km}$ track (Jayawant, 1988). Recent discoveries of new superconducting materials may mean that, in the future, this will be the more successful system but, at present, the EMS system presents the better option. EMS systems used the attractive force of sets of electromagnets acting upwards towards steel tracks to levitate the vehicle. The most advanced EMS system is at Emsland in Germany, where speeds of $400 \mathrm{~km} \mathrm{hr}^{-1}$ have been reached on a $31.5-\mathrm{km}$ test track, whilst Japanese Air Lines had a $55 \mathrm{~km} \mathrm{hr}^{-1}$ system operating on a $400-\mathrm{m}$ track at Tsukuba Expo 85. In the U.K., a low velocity 8-ton commercial passenger-carrying system has been implemented over a 620-m track at Birmingham Airport (Sinha, 1987).

The electro-magnetic suspension of a vehicle is inherently unstable, and thus active control is required. In addition, it is necessary to maintain the airgap between the supporting guideway and the electromagnetics in order to prevent undesirable contact between them. Systems where the error or other outputs are required to be strictly within bounds are known as 'critical' systems (Zakian, 1989) and the control of a maglev suspension system is one such system. Control systems are subject to external inputs and disturbances. The aim of the designer of a critical system is to ensure that the

* Received 12 January 1989; revised 15 February 1990; revised 10 June 1991; revised 10 December 1992; received in final form 6 January 1993. The original version of this paper was not presented at any IFAC meeting. This paper was recommended for publication in revised form by Associate Editor G. Verghese under the direction of Editor H. Austin Spang III.

$\dagger$ Control Systems Research, Department of Engineering, University of Leicester, Leicester LE1 7RH, U.K. FAX (44) 533 522619; e-mail jfw@uk.ac.le.engg.sun. inputs do not disturb the system beyond the critical level, thereby making the system tolerant to the inputs. Ideally a system should be designed so that the set of inputs to which it is subjected is the same as the set to which it is tolerant. Such a system is said to be perfectly well-matched to its environment (Zakian, 1991).

The problem of critical systems has been recognized in other works, and various methods have been suggested to cope with it. Statistical criteria for the design of critical systems are described by Andreyev (1969), and are based on maximizing the probability that the outputs will remain within the desired bounds when subjected to random inputs, the inputs being described in terms of probability distributions. Another method is the set theoretic approach (Glover and Schweppe, 1971; Bertsekas and Rhodes, 1971; Schweppe, 1973) which introduces the concept of a "target tube', within which the system states or outputs must be maintained whilst subjected to unknown-but-bounded disturbances. A computationally efficient algorithm for designing control systems using the set theoretic approach has been developed (Usoro et al., 1982) and successfully applied to a control system (Parlos et al., 1988).

Zakian's design framework (Zakian, 1984, 1986a, 1986b, $1987 \mathrm{a}, 1989,1991)$ is very well suited for the design of critical systems. The designer can specify bounds on one or more of the outputs. Also, a wide set of inputs may be considered, including persistent and transient inputs, and increases the likelihood that the designer can design a well-matched system. This paper is devoted to the initial design of the EMS control system of a high-speed maglev vehicle, using Zakian's framework

2. Zakian's design framework (Zakian, 1984, 1986a, 1986b, $1987,1989,1991$ )

A critical system, subject to a single input and with one output to be bounded, has to satisfy the inequality

$$
|v(t, f, p)| \leq \varepsilon, \quad \text { for all } t \in \mathbb{R},
$$

where $v(t, f, p)$ is the system output, usually the error, $t$ is time, $f$ is an input which maps a set of real numbers into itself, $p$ is a controller chosen from a given set $P$ and $\varepsilon$ is the prescribed bound that must not be exceeded by the system output.

Suppose that the input is known only to the extent that it belongs to the known function space $\mathscr{F}_{D}$ defined as the class of all functions $f: \mathbb{R} \rightarrow \mathbb{B}$ such that $f(t)=0$ for all $t \leq 0$, and the derivative $f^{(i)}$ is piecewise continuous and, for some $0<D<\infty$

$$
\left|f^{(1)}(t)\right| \leq D, \quad \text { for all } t \in \mathbb{R} .
$$

The input space is here defined by a bound on the derivative of the input. This is because it has been shown (Zakian, 1986a) that an input space defined only by a bound on the magnitude of the input may lead to errors that are uncontrollable, thus making the control problem meaningless. 
Suppose that for all $f \in \mathscr{F}_{D}$ the output is related to the input by

$$
v(t, f, p)=\int_{0}^{t} v(t-\lambda, h, p) f^{(1)}(\lambda) \mathrm{d} \lambda,
$$

where the unit step response $v(t, h, p)$ is piecewise continuous, then (Zakian, 1987)

$$
\hat{v}(p)=\sup \left\{|v(t, f, p)|: t \in \mathbb{R}, f \in \mathscr{F}_{D}\right\} .
$$

where

$$
\hat{v}(p)=D \int_{0}^{\infty}|v(t, h, p)| \mathrm{d} t,
$$

The design problem can be written

$$
\hat{v}(p) \leq \varepsilon \text {. }
$$

Clearly if inequality (6) holds, the performance of the critical system is guaranteed, i.e. (1) holds for all $f \in \mathscr{F}_{D}$. Computational procedures exist to solve (6).

In order that (6) can be solved using numerical methods, the inequality

$$
\hat{v}(p)<\infty
$$

must first be solved. If the transfer function from $f$ to $v(f)$ is rational, it can be shown (Zakian, 1986a) that (7) holds if and only if

$$
\begin{gathered}
\lim _{s \rightarrow 0} s V(s, p)=0, \\
\operatorname{Re}\{\lambda(p)\}<0, \text { for all } \lambda(p) \in \Lambda(p),
\end{gathered}
$$

where $V(s, p)$ is the Laplace transform of $v(t, h, p)$ and $\Lambda(p)$ denotes the set of all the finite poles $\lambda(p)$ of $V(s, p)$. Here, $\operatorname{Re}\{x\}$ denotes the real part of a complex number $x$.

The results (4), (8) and (9), for the single-input single-output case can be extended to the maglev suspension system which has three outputs to be bounded, and an input consisting of the sum of two inputs. Accordingly, let

$$
f=f_{1}+f_{2},
$$

with input $f_{1}$ belonging to the input space $\mathscr{F}_{D}$ defined as in (2) with a corresponding bound $D_{1}$. The second input $f_{2}$ is known only to the extent that it belongs to the known function space $\mathscr{F}_{A}$ defined as the class of all functions $f_{2}: \mathbb{R} \rightarrow \mathbb{R}$ such that $f_{2}(t)=0$ and $f_{2}^{(1)}(t)=0$ for all $t \leq 0$, and the second derivative $f_{2}^{(2)}$ is piecewise continuous and, for some $0<D<\infty$

$$
\left|f_{2}^{(2)}(t)\right| \leq D_{2}, \quad \text { for all } t \in \mathbb{R} .
$$

The input space is here defined by a bound on the second derivative of the input.

The outputs are denoted by $v_{i}(t, f, p)$ for $i=1,2,3$. For all $f_{1} \in \mathscr{F}_{D}$ and for all $f_{2} \in \mathscr{F}_{A}$, the outputs are related to the input by

$$
\begin{aligned}
v_{i}(t, f, p)= & \int_{0}^{t} v_{i}(t-\lambda, h, p) f_{1}^{(1)}(\lambda) \mathrm{d} \lambda \\
& +\int_{0}^{t} v_{i}(t-\lambda, r, p) f_{2}^{(2)}(\lambda) \mathrm{d} \lambda, \quad i=1,2,3,
\end{aligned}
$$

where the unit step responses $v_{i}(t, h, p)$ and the unit ramp responses $v_{i}(t, r, p)$ are piecewise continuous $\left(v_{i}(t, r, p)\right.$ are the integrals of the unit step responses $\left.v_{i}(t, h, p)\right)$. Following from Zakian (1986a, 1987),

$$
\hat{v}_{i}(p)=\sup \left\{\left|v_{i}(t, f, p)\right|: t \in \mathbb{R}, f_{1} \in \mathscr{F}_{D}, f_{2} \in \mathscr{F}_{A}\right\} .
$$

where

$$
\begin{aligned}
\hat{v}_{i}(p)= & D_{1} \int_{0}^{\infty}\left|v_{i}(t, h, p)\right| \mathrm{d} t \\
& +D_{2} \int_{0}^{\infty}\left|v_{i}(t, r, p)\right| \mathrm{d} t, \quad i=1,2,3,
\end{aligned}
$$

The design criteria are expressed by

$$
\hat{v}_{i}(p) \leqslant \varepsilon_{i}, \quad i=1,2,3,
$$

where $\varepsilon_{i}$ is the largest tolerable value of $\left|v_{i}(t, f, p)\right|$.
In order for

$$
\hat{v}_{i}(p)<x, \quad i=1,2,3,
$$

the following conditions are necessary and sufficient

$$
\begin{gathered}
\lim _{s \rightarrow 0} V_{i}(s, p)=0, \text { for } i=1,2,3, \\
\operatorname{Re}\left\{\lambda_{i}(p)\right\}<0, \text { for all } \lambda_{i}(p) \in \Lambda_{i}(p) \text { for } i=1,2,3,
\end{gathered}
$$

where $V_{i}(s, p)$ is the Laplace transform of $v_{i}(t, h, p)$ and $\Lambda_{i}(p)$ denotes the set of all the finite poles $\lambda_{i}(p)$ of $V_{i}(s, p)$.

The set of inequalities (15) may be solved by means of computational procedures such as the method of inequalities (Zakian and Al-Naib, 1973; Maciejowski, 1989; Ng, 1989). Various other procedures may also be used (Macicjowski, $1989 ; \mathrm{Ng}, 1989)$. The method of inequalities requires that a multiobjective problem be formulated as a set of inequalities and contains an iterative design procedure, the object of which is to satisfy the set of inequalities. The procedure is interactive, in that it requires supervision and intervention from the user. It involves choosing the controller structure, the starting point of the search algorithm, and appropriately adjusting the bounds $\varepsilon_{i}$. The method of inequalities has been incorporated into the CAD suite, 'Criteria' (Bada, 1987a), and the procedure is summarized below.

(i) Define the plant model and parameters, the parameters $D_{1}$ and $D_{2}$ of the input spaces $\mathscr{F}_{I}$ and $\mathscr{F}_{A}$ and the bounds $\varepsilon_{i}$.

(ii) Choose the structure of the controller, which also determines the dimension $N$ of vector $p$ and define the bounds on the elements of $p$.

(iii) Choose the starting point $p \in \mathbb{B}^{N}$

(iv) Implement a search algorithm, such as the moving boundaries process (Zakian and Al-Naib, 1973) to locate a stability point, i.e. a point $p$ such that $(18)$ is satisfied.

(v) Starting with a stability point, use the search algorithm to locate a point $p$ which satisfies inequalities (15) and other constraints such as the bounds on the elements of $p$

The interactive nature of the algorithm provides the user with great flexibility. If a solution is not found easily, the designer can change the starting point to avoid computational traps (such as a local minimum of $\hat{v}_{i}(p)$ ), increase the complexity of the controller or, if appropriate, relax one or more of the bounds $\varepsilon_{i}$. Alternatively, if a successful design is easily located, and $\hat{v}_{i}(p)$ are well within the specified bounds $\varepsilon_{i}$, the designer may decrease the controller complexity, so giving a cheaper implementation, or may tighten some of the bounds $\varepsilon_{i}$ to obtain a higher quality system.

3. Design of an electro-magnetic suspension control system

Recent rapid advances in solid state electronics have meant that electro-magnetically levitated transport systems are now a feasible option. Dynamic control is required for the vertical lift system in order to give stability to an inherently unstable system, to maintain the airgap and to ensure the quality of the ride for the passengers. The necessity to maintain the airgap to prevent contact between the vehicle and the guideway means that the vertical control system is a critical system, and this section describes how this critical control system is designed using Zakian's framework.

The design considered here is an initial design using a linearized one-dimensional model, considering just the vertical heave dynamics. The method could similarly be applied to a more complex three-dimensional model, which would include pitch, roll and yaw dynamics along with lateral and vertical heave dynamics. The model is for a vehicle consisting of a chassis supporting a passenger cabin by means of a secondary suspension consisting of airsprings and hydraulic shock absorbers. The chassis is levitated by means of dc electromagnets under active control providing an attractive force to the guideway. The cross-section of the vehicle showing the configuration is given in Fig. 1. The vehicle is also equipped with emergency skids in case of power failure. 


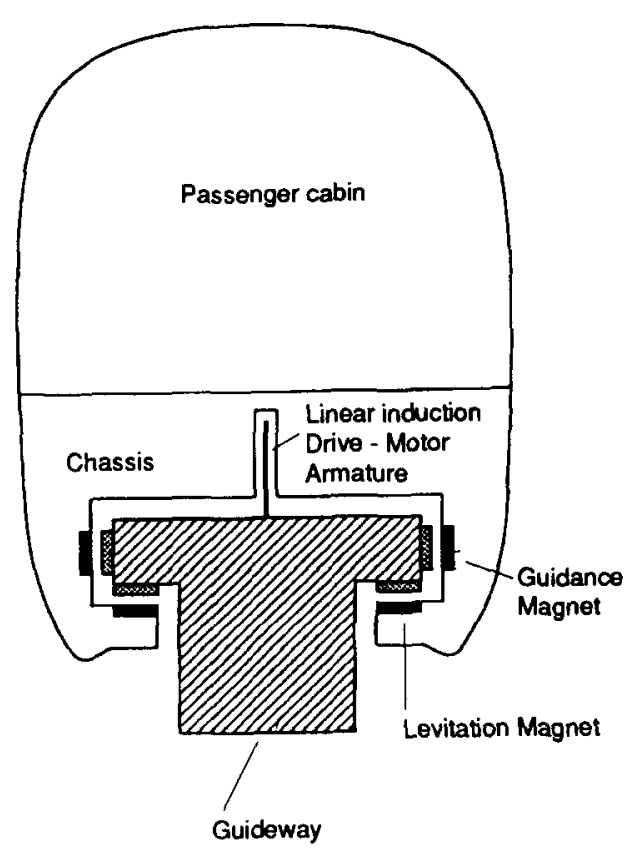

Fig. 1. Maglev vehicle cross-section.

The one-dimensional design model (Kortüm and Utzt, 1984; Müller, 1977) considers the vertical movement of the chassis and passenger cabin. The secondary suspension is modeled as a linear spring damper system, and the primary electro-magnetic suspension is described by a linear first order differential equation for the vertical force being derived from the magnet force law and the current/voltage relation. The model configuration is shown in Fig. 2.

The chassis has mass $m_{1}$ and the passenger cabin has mass $m_{2}$. The secondary suspension has a spring constant $c$ and damping constant $d$. The relationship between the two is assumed linear and satisfies Newton's law:

$$
\begin{aligned}
& m_{1} x_{1}^{(2)}(t)+d\left(x_{1}^{(1)}(t)-x_{2}^{(1)}(t)\right)+c\left(x_{1}(t)-x_{2}(t)\right)=P_{m}(t) \\
& m_{2} x_{2}^{(2)}(t)+d\left(x_{2}^{(1)}(t)-x_{1}^{(1)}(t)\right)+c\left(x_{2}(t)-x_{1}(t)\right)=0
\end{aligned}
$$

where $x_{1}$ is the absolute position of the chassis, and $x_{2}$ is the absolute position of the passenger cabin. $P_{m}$ is the force exerted by the electromagnet beyond that required to overcome gravity

$$
P_{m}(t)=K_{1} i(t)-K_{2} z(t) .
$$

The electric current $i$ is related to the applied voltage $u$ by

$$
u(t)=R i(t)+L i^{(1)}(t)-K_{3} z^{(1)}(t) .
$$

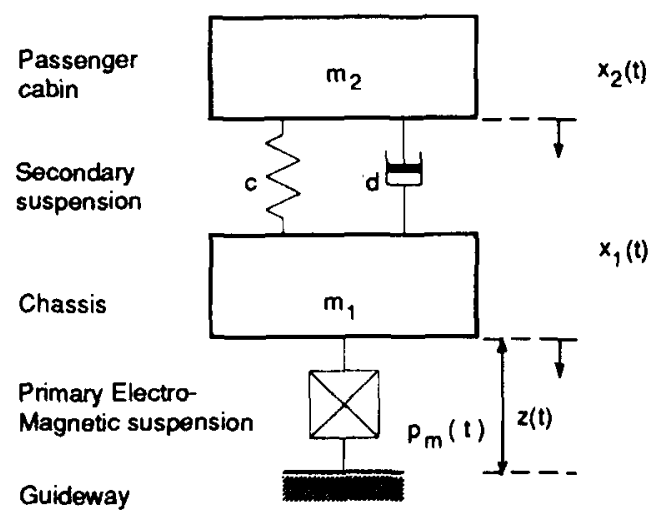

FIG. 2. Maglev vehicle model configuration.

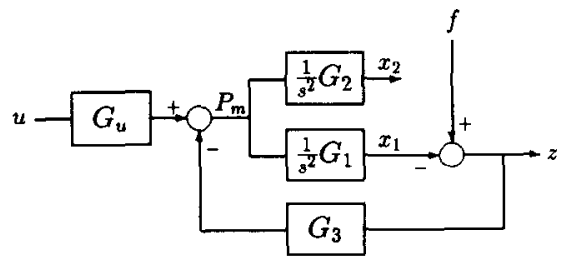

FIG. 3. System configuration with guideway disturbance $f$.

where

$$
z(t)=f(t)-x_{1}(t)
$$

is the gap width, $f(t)$ being the disturbance resulting from variations in the guideway profile. The relations (21) and (22) are linearized with respect to an equilibrium position-the variables represent deviations about their steady-state equilibrium values.

Typical values of the constants are (Müller, 1977)

$$
\begin{aligned}
K_{1} & =580 \mathrm{~N} \mathrm{~A}^{-1}, \\
K_{2} & =1.3 \times 10^{6} \mathrm{~N} \mathrm{~m}^{-1}, \\
K_{3} & =580 \mathrm{Vsec} \mathrm{m}^{-1}, \\
L & =0.516 \mathrm{Vsec} \mathrm{A}^{-1}, \\
R & =20 \mathrm{hms}, \\
m_{1} & =500 \mathrm{~kg}, \\
m_{2} & =500 \mathrm{~kg}, \\
c & =10^{5} \mathrm{~N} \mathrm{~m}^{-1}, \\
d & =10^{4} \mathrm{Nsec} \mathrm{m}^{-1} .
\end{aligned}
$$

The system configuration with the guideway disturbance is shown in Fig. 3, where from (19) to (23)

$$
\begin{gathered}
G_{u}(s)=\frac{580}{0.516 s+2} \mathrm{~N} \mathrm{~V}^{-1}, \\
G_{1}(s)=\frac{s^{2}+20 s+200}{500\left(s^{2}+40 s+400\right)} \mathrm{m} \mathrm{N}^{-1} \sec ^{-2}, \\
G_{2}(s)=\frac{(s+10)}{25\left(s^{2}+40 s+400\right)} \mathrm{m} \mathrm{N}^{-1} \sec ^{-2} \\
G_{3}(s)=\frac{334400 s+2600000}{0.516 s+2} \mathrm{~N} \mathrm{~m}^{-1}
\end{gathered}
$$

The major disturbance to the system is from variations in the guideway height. These variations arise from two sources, the geometry of the guideway can thus be represented by

$$
f(t)=f_{1}(t)+f_{2}(t),
$$

where $f_{2}(t)$ represents the guideway variations due to gradients in the terrain, and $f_{1}(t)$ are irregularities in the guideway, caused mainly by sagging between the guideway supports as well as by maladjustments in the rail and thermal effects. The following bounds for the guideway have been suggested for a $140-\mathrm{m} \mathrm{sec}^{-1}$ vehicle (Müller, 1977):

$$
\sup \left\{\left|f_{1}^{(1)}(t)\right|: t \geq 0\right\}=0.030 \mathrm{~m} \mathrm{sec}^{-1}
$$

and

$$
\sup \left\{\left|f_{2}^{(2)}(t)\right|: t \geq 0\right\}=0.100 \mathrm{~m} \mathrm{sec}^{-2} .
$$

Thus from (2) and (11), the two input spaces are characterized by

$$
D_{1}=0.030 \mathrm{~m} \mathrm{sec}^{-1}
$$

and

$$
D_{2}=0.100 \mathrm{~m} \mathrm{sec}^{-2} \text {. }
$$

The rate bound on $f_{1}$ reflects the quality of the guideway, and the acceleration bound on $f_{2}$ represents a restriction on the suddenness with which a gradient begins. 
The primary purpose of the vertical control system is to provide stability to an otherwise unstable system. An electromagnet excited by a constant voltage will either clamp to the rail or fall because the attractive force decreases with increasing airgap. Once stability has been ensured by the control scheme there are two important outputs which have to be considered in determining the effectiveness of the control system

(i) It is desirable to avoid contact between the guideway and the skids in order that wear of and possible damage to the guideway and skids and minimized, and to avoid unnecessary vibration, friction and noise. In addition, frequent contact leads to a deterioration in the ride quality. This means that the control system is critical, in that the minimum permissible airgap has a definite bound. The allowed airgap is between 7 and $17 \mathrm{~mm}$ which means a nominal gap of $12 \mathrm{~mm}$. A minimal gap of $7 \mathrm{~mm}$ assures a sufficient safety margin to allow for the failure of single magnets (Kortüm and Utzt, 1984). The nominal airgap cannot be too large as lifting capacity decreases with growing airgap, and thus the nominal airgap must be limited in order to obtain reasonable magnet weights and power consumption. Thus, the maximum error permitted between the airgap and the nominal airgap is $5 \mathrm{~mm}$.

(ii) The ride comfort can be determined by the amount of vertical acceleration experienced by the passengers. Thus it is important that the maximum acceleration in either direction is not too great. Typically, this should not exceed $0.5 \mathrm{~m} \mathrm{sec}^{-2}$ (Kortüm and Utzt, 1984). This second criterion is important, but is not rigid and may be increased to as much as $1.0 \mathrm{~m} \mathrm{sec}^{-2}$ (Armstrong, 1984).

(iii) An additional criterion is in the amount of control effort required: the required control voltage should be within -600 and $+6(0) \vee$ (Kortüm and Utzt, 1984).

The design criteria (15) is rewritten

$$
\hat{v}_{i} \leq \varepsilon_{i}, \quad i=1,2,3,
$$

where

$$
\begin{aligned}
& v_{1}=e, \\
& v_{2}=x_{2}^{(2)}, \\
& v_{3}=u,
\end{aligned}
$$

and

$$
\begin{aligned}
\varepsilon_{1} & =5 \mathrm{~mm}, \\
\varepsilon_{2} & =500 \mathrm{~mm} \mathrm{sec}{ }^{2} \\
\varepsilon_{3} & =600 \mathrm{~V} .
\end{aligned}
$$

Thus with the design criteria specified, and the plant and the input spaces determined, relationship (13) was used with the 'Criteria' package implemented on a Sun Workstation to determine a set of controllers that would satisfy the criteria.

An inductive sensor is used to measure the gap width $z$, and an accelerometer for the chassis acceleration $x_{1}^{(2)}$. These two signals are used to implement a feedback control scheme as shown in Fig. 4.

The presence of a double integrator in the plant means that condition (17) is satisfied, so the first stage was to satisfy (18), i.e. stabilize the system, and this was achieved after only a few iterations. The next stage was to satisfy (34). After a number of iterations, the criteria (34) had not been satisfied, so the bound $\varepsilon_{2}$ on the passenger acceleration $x_{2}^{(2)}$ was relaxed in stages until

$$
\begin{aligned}
& \varepsilon_{1}=5 \mathrm{~mm}, \\
& \varepsilon_{2}=800 \mathrm{~mm} \mathrm{sec}-2, \\
& \varepsilon_{3}=600 \mathrm{~V} .
\end{aligned}
$$

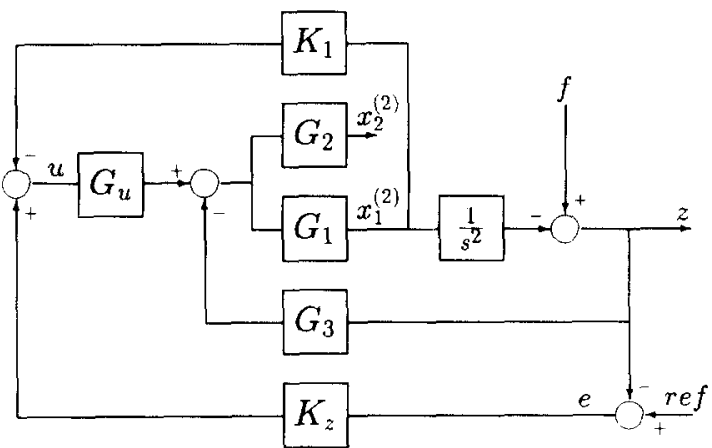

FIG. 4. Maglev vehicle suspension control scheme

With these bounds the criteria (34) are satisfied by

$$
\begin{gathered}
K_{z}=-1 \times 10^{6} \frac{1.7 s^{2}+10.5 s+31.5}{0.05 s^{2}+20 s+1}, \\
K_{1}=1 \times 10^{6} \frac{2.22 s^{2}+16.0 s+0.73}{648 s^{2}+61 s+1},
\end{gathered}
$$

giving

$$
\begin{aligned}
& \hat{v}_{1}=4.81 \mathrm{~mm}, \\
& \hat{v}_{2}=800 \mathrm{~mm} \mathrm{sec}^{-2}, \\
& \hat{v}_{3}=238 \mathrm{~V} .
\end{aligned}
$$

The bound $\varepsilon_{2}$ is not rigid, the consequence of it being increased is a lower level of passenger comfort. However $\hat{v}_{2}$ represents the greatest possible acceleration of the passenger carriage, and may only occur rarely; hence this design is acceptable.

A typical guideway disturbance is shown in Fig. 5. Input $f_{1}(t)$ is a constant gradient of $30 \mathrm{~mm} \mathrm{sec}^{-1}$ followed by a sinusoid with a maximum gradient of $30 \mathrm{~mm} \mathrm{sec}^{-1}$ and $f_{2}(t)$ is a curve with $f_{2}^{(2)}(t)=100 \mathrm{~mm} \mathrm{sec}{ }^{-2}$ followed by a constant gradient. The summed input $f(t)$ therefore represents a severe test of the system. Responses to this input are shown in Figs 6-8, and it can be seen from these that bounds (41)-(43) are not exceeded.

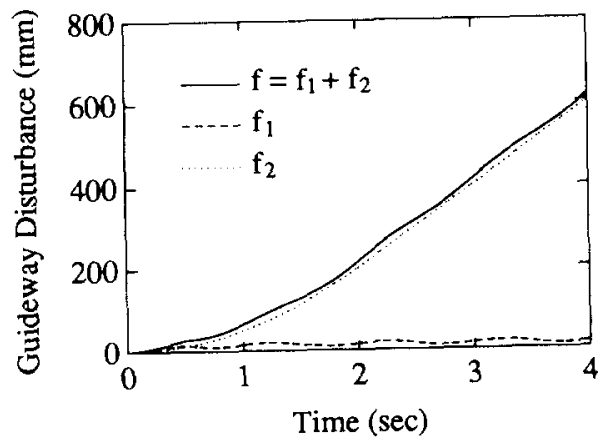

FIG. 5. Typical input.

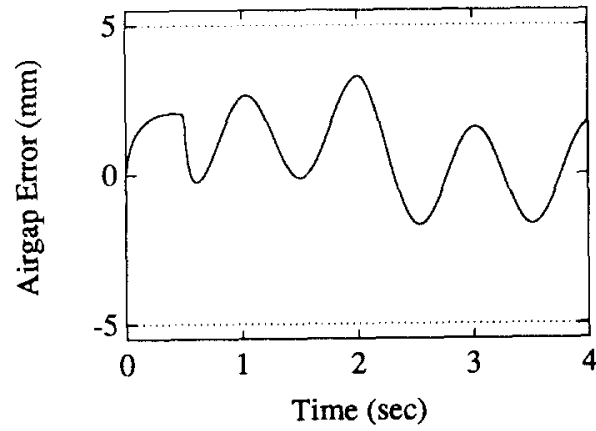

FIG. 6. Airgap error response to typical input shown to stay within the bounds $\varepsilon_{1}=5$. 


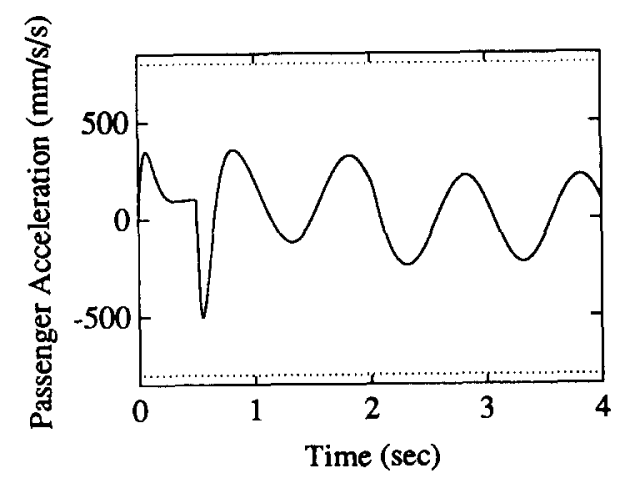

Fig. 7. Passenger carriage acceleration response to typical input shown to stay within the bounds $\varepsilon_{2}=800$.

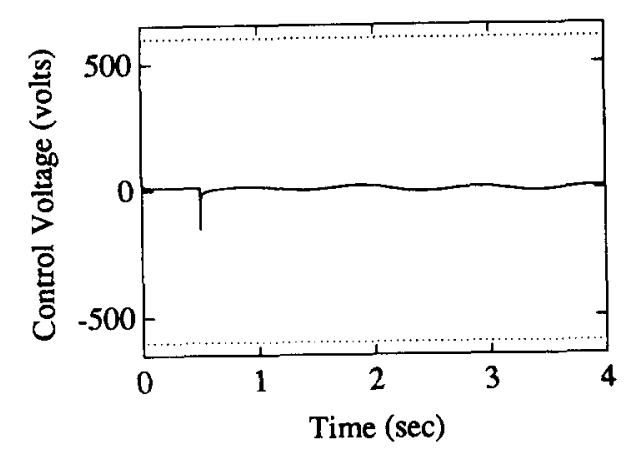

FIG. 8. Control voltage response to typical input shown to stay within the bounds $\varepsilon_{2}=600$.

\section{Conclusions}

The suspension control of a maglev vehicle is a critical system in that it is necessary to maintain the airgap whilst keeping the passengers adequately comfortable and whilst not exceeding the maximal control voltage. The majo disturbance is caused by variations in the guideway profile. disturbance is caused from two sources, variations in the terrain and guideway irregularities. These variations will typically have bounds on their derivatives, and can thus be characterized by input spaces provided in Zakian's framework. Zakian's design framework provides an effective method of designing critical systems with a set of tolerable inputs which are well-matched to the disturbances which occur in practice. The framework was hence used to obtain an effective controller design using a simplified onedimensional linear model of the electro-magnetic suspension.

Zakian's method could be similarly used to design a more complex three-dimensional model of a maglev vehicle. In addition, variations of the model parameters do occur, resulting from non-linearities in the system. A more comprehensive design would require these variations to be taken into account. The theoretical basis for this is provided by Zakian (1984), and the method has been successfully applied (Taiwo, 1986; Bada, 1987b).

The application of Zakian's method for this particular design is closely related to the $L^{1}$-optimal control approach (Dahleh and Pearson, 1987). However, the $L^{\prime}$-optimal approach produces controllers which are not rational and therefore more difficult to implement. The most useful application of the $L^{\prime}$-optimal approach may be in providing the limit of possible performance; thus, if the minimum possible $\hat{v}$ is greater than $\varepsilon$, there is no solution to the critical problem and it has to be reformulated. The set theoretic approach (Glover and Schweppe, 1971; Bertsekas and Rhodes, 1971; Schweppe, 1973) can also be used to design critical systems, and a systematic study to compare the relative merits of Zakian's and the set theoretic approach would be worth undertaking.

Acknowledgements-The author is grateful to Dr V. Zakian for his advice and guidance and to the reviewers for their helpful comments and suggestions.

\section{References}

Andreyev, N. I. (1969). Correlation Theory of Statistically Optimal Systems. W. B. Saunders, Philadelphia, PA.

Armstrong, D. S. (1984). Magnet/rail systems-a critical review of the options. Proc. Int. Mech. Eng. Conf. on Maglev Transport Now and for the Future, Solihull, U.K., pp. 59-66.

Bada, A. T. (1987a). CRITERIA-a package for computeraided control system design. Computer-aided Design, 19, 466-474.

Bada, A. T. (1987b). Robust brake control for a heavy-duty truck, IEE Proc. D, 134, 1-8.

Bertsekas, D. P. and I. B. Rhodes (1971). On the minimax reachability of target sets and target tubes. Automatica, 7 , 233-247.

Dahleh, M. A. and J. B. Pearson (1987). L'-optimal compensators for continuous-time systems. IEEE Trans. Aut. Control, 32, 889-895.

Glover, J. D. and F. C. Schweppe (1971). Control of linear dynamic systems with set constrained disturbances. IEEE Trans. Aut. Control, 16, 411-422.

Jayawant, B. V. (1988). Are we in control? IEE Proc. D, 135, $1-15$.

Kortüm, W. and A. Utzt (1984). Control law design and dynamic evaluations for a maglev vehicle with a combined lift and guidance suspension system. ASME J. Dynamic Sys. Measurement and Control, 106, 286-292.

Maciejowski, J. M. (1989). Multivariable Feedback Design, pp. 341-346. Addison-Wesley, Wokingham, U.K.

Müller, P. C. (1977). Design of optimal state-observers and its application to maglev vehicle suspension control. Proc. 4th IFAC Symp. Multivariable Technological Systems, Fredericton, Canada, pp. 175-182.

$\mathrm{Ng}$, W.-Y. (1989). Interactive multi-objective programming as a framework for computer-aided control system design. Lecture Notes in Control and Information Sciences, Vol. 132. Springer-Verlag, Berlin.

Parlos, A. G., A. F. Henry, F. C. Schweppe, L. A. Gould and D. D. Lanning (1988). Non-linear multivariable control of nuclear power plants based on the unknownbut-bounded disturbance model. IEEE Trans. Aut. Control, 33, 130-137.

Schweppe, F. C. (1973). Uncertain Dynamical Systems. Prentice-Hall, Englewood Cliffs, NJ.

Sinha, P. K. (1987). Electromagnetic Suspensions: Dynamics and Control. Peter Peregrinus, London.

Taiwo, O. (1986). The design of robust control systems for plants wth recycle. Int. J. Control, 43, 671-678.

Usoro, P. B., F. C. Schweppe, D. N. Wormley and L. A. Gould (1982). Ellipsoidal set-theoretic control synthesis. ASME J. Dynamic Sys. Measurement and Control, 104, 331-336.

Zakian, V. (1984). A framework for design: theory of majorants. Report No. 604, Control Systems Centre, UMIST, Manchester, U.K.

Zakian, V. (1986a). A performance criterion. Int. J. Control, 43, 921-931.

Zakian, V. (1986b). On performance criteria. Int. J. Control, 43, 1089-1092.

Zakian, V. (1987). Input spaces and output performance. Int. J. Control, 46, 185-191.

Zakian, V. (1989). Critical systems and tolerable inputs. Int. J. Control, 49, 1285-1289.

Zakian, V. (1991). Well matched systems. IMA J. Math. Control Inf., 8, 29-38.

Zakian, V. and U. Al-Naib (1973). Design of dynamical and control systems by the method of inequalities. Proc. IEE, 120, 1421-1427. 
1993-09-01

\section{EMS control system design for a Maglev vehicle - a critical system}

Whidborne, James F.

Elsevier

pÿJ.F. Whidborne, EMS control system design for a maglev vehicle A critical system, Automatica, Volume 29, Issue 5, 1993, Pages 1345-1349

http://dx.doi.org/10.1016/0005-1098(93)90054-W

Downloaded from Cranfield Library Services E-Repository 